\title{
Consumo de noticias y temor al delito en Chile
}

\author{
Andrés Scherman TEITELBOIM \\ Universidad Diego Portales (Chile) \\ andres.scherman@udp.cl \\ Nicolle ETCHEGARAY THIELEMANN \\ Universidad Diego Portales (Chile) \\ nicole.etchegaray@udp.cl
}

Recibido: 03/07/2012

Aceptado: 21/11/2012

\begin{abstract}
Resumen
Chile es uno de los países con menores niveles de criminalidad en América Latina, pero exhibe altos niveles de temor. Esta investigación examina la influencia del consumo medial, entre otros factores, como determinante de esta dicotomía. Una serie de análisis de regresión aplicados a los datos de una encuesta probabilística, permiten concluir que existe una relación positiva entre el consumo de noticias en televisión abierta y el miedo a ser víctima de delito. También se observa que comentar noticias sobre delincuencia tiene un impacto relevante sobre los niveles de temor. Sin embargo, las variables que más influyen en el temor no son las vinculadas a los medios, sino otras como la percepción de seguridad del entorno, sexo, edad y haber sido víctima de un delito.
\end{abstract}

Palabras clave: Temor al delito, medios de comunicación social, noticias, efectos televisivos, agenda.

\section{News and Fear of Crime in Chile}

\begin{abstract}
Despite the fact that Chile is one of the countries with the lowest crime rates in Latin America, its population exhibits high levels of fear of crime. This research examines the influence of media consumption, among other factors, as a determinant of the fear of crime. A series of regression analysis indicate a positive relationship between an individual's consumption of news television and fear of being a victim of crime. The study also finds that news commentary on crime has a significant impact on levels of fear. Nevertheless, the variables with the greatest impact on fear are not related to the media, but include perceptions on the environment problems, the respondent's gender, age and whether he or she has been the victim of a crime.
\end{abstract}

Keywords: Fear of crime, mass media, news, television influence, agenda.

\section{Referencia normalizada}

SCHERMAN TEITELBOIM, Andrés y ETCHEGARAY THIELEMANN, Nicolle (2013): "Consumo de noticias y temor al delito en Chile". Estudios sobre el mensaje periodístico. Vol. 19, Núm. 1, págs.: 563-575. Madrid, Servicio de Publicaciones de la Universidad Complutense.

Sumario: 1. Introducción. 2. Fuentes y Metodología. 3. Desarrollo. 4. Conclusiones. 5. Referencias bibliográficas

\section{Introducción}

La seguridad ciudadana es uno de los principales temas de debate público en América Latina. Según la Corporación Latinobarómetro, ante la pregunta abierta sobre el problema más importante, la respuesta mayoritaria el año 2011 fue la delincuencia y la seguridad pública (28\%).

En Chile, uno de los países con menos violencia en la región (Lapop, 2010), la delincuencia es la principal preocupación de la ciudadanía. De acuerdo a sondeos de 
opinión, un $47 \%$ de la población considera que el problema más importante que el gobierno debe solucionar es "delincuencia, asaltos y robos" (Centro de Estudios Públicos, 2011), y aunque sólo el 38,6\% de los chilenos declaraba haber sido víctima de delitos, el 86,1\% presentaba niveles de temor "medio" o "alto" (Fundación Paz Ciudadana-AdimarkGfk, 2012).

Los datos comprueban que la relación entre miedo y realidad no es directa. En numerosos casos, como en Chile, a bajas sostenidas en las tasas de criminalidad, le corresponde el mantenimiento de los niveles de temor y preocupación por la seguridad.

De acuerdo al Índice Paz Ciudadana-Adimark Gfk, la victimización en Chile disminuyó desde 37,9 a 34,2\% entre 2005 y 2010 (2010). Según estudios del gobierno (Ministerio del Interior y Seguridad Pública, 2010) los hogares víctimas bajaron desde un 38,3 a un $28,2 \%$ en el mismo periodo. Sin embargo, durante la década el porcentaje de hogares que se ubica en un nivel de temor "alto" creció de manera importante, alcanzando su mayor nivel en 2007, para luego comenzar a disminuir. En 2010, el $70,9 \%$ de la población afirmaba que la delincuencia afectaba mucho su calidad de vida (Fundación Paz Ciudadana, 2010).

El rol de los medios de comunicación sobre esta "percepción aumentada" de temor es un debate no resuelto. Las investigaciones destinadas a determinarlo coinciden en la tendencia de los medios a sobrerepresentar la realidad del delito y la violencia, pero hay conclusiones disímiles en torno a las consecuencias de esta situación en la percepción de la realidad.

La teoría de Agenda Setting, McCombs plantea que los medios ejercen su mayor influencia estableciendo los temas que capturan la atención de la ciudadanía, entre ellos el crimen y la violencia delictual (McCombs, 1996). Así, una programación o cobertura noticiosa centrada en el crimen, no tendría tanto eco en la percepción específica de temor, como en la valoración del tema como uno relevante dentro de las preocupaciones públicas.

De acuerdo a la "teoría del cultivo", en cambio, los medios de comunicación socializan o "cultivan" a sus audiencias en una visión del mundo, implantando valores comunes e influyendo en las creencias sobre ésta, así como en los estados cognitivos y emocionales. De acuerdo a sus principales exponentes (Gerbner et al., 1980), el temor es un subproducto de la alta exposición de la ciudadanía a una programación medial violenta y noticiarios enfocados en la difusión de delitos. El nivel de influencia, según esta teoría, está ligado a la intensidad de la exposición a los medios y a la selección de determinados espacios y contenidos. Ante mayor consumo de contenidos que ofrecen una imagen de inseguridad y violencia, mayor es la capacidad de influir en la sensación de temor.

Sin embargo, la investigación empírica ha ofrecido contradictoria evidencia sobre la validez de esta sentencia. La investigación de Stutman (1995) plantea que, lejos del temor, la prolongada exposición a temáticas violentas en los medios produce acostumbramiento, una suerte de insensibilización emocional frente a la violencia.

La investigación sobre la influencia de los medios en la percepción de temor es mayoritariamente norteamericana y cuantitativa. Su historia ha evolucionado desde los estudios sobre los supuestos "efectos" persuasivos de los medios, hacia una concep- 
ción centrada en los "usos" que las audiencias dan a éstos, en función de diferentes variables, como el contexto económico y socio-cultural que, sin lugar a dudas, juega un papel muy importante en el caso del miedo y la violencia.

Un estudio del Departamento de Justicia Criminal de la Universidad de California (Dowler, 2003) mostró que las personas que son espectadoras regulares de programación sobre crimen son más propensas a temer a la delincuencia, aunque son otros los factores estadísticamente más relacionados con el miedo.

Otras investigaciones reafirman que la sensación de temor está determinada por la conjunción de diversos factores, que generan modelos complejos de análisis. Se plantea que son variables relevantes la propia experiencia en torno al crimen (victimización previa, directa o vicaria), la percepción de seguridad en el entorno, el género, el estrato socio-económico, el nivel de confianza en las instituciones, el nivel de confianza interpersonal y las imágenes de la realidad proyectadas por los medios de comunicación en torno a la temática (Gerbner, 1980; Ditton, 2004; Reiner, 2007).

En Chile existe escasa investigación empírica relacionada. A través de técnicas de análisis de contenido, se ha comprobado la sobrerrepresentación de la realidad delictual en los medios de comunicación nacionales (Dammert, 2005; Browne y Tomicic, 2007). Sin embargo, no se ha realizado un acercamiento a las audiencias para analizar la influencia de los medios en el temor. Sólo una investigación exploró esta relación (Allende, 2004) y concluyó que no es posible sostener que existe una relación estadísticamente significativa entre el consumo de medios y el temor; sin embargo, se trata de una investigación orientada a determinar de forma amplia los elementos determinantes del temor a la delincuencia y sólo incorpora la exposición a los medios a través de la variable "atención a noticias delictuales en televisión", sin considerar aspectos como el uso de otros medios de comunicación, el consumo de programas específicos sobre delincuencia o las conversaciones interpersonales que puede generar los medios.

Una investigación de Browne y Tomicic, basada en los mismos datos utilizados por Allende, muestra que la atención a noticias delictuales en televisión es un factor determinante de tener un alto temor a ser robado o asaltado. Aunque las autores cuestionan si realmente se puede establecer una relación causal entre ambos fenómenos (Browne y Tomicic, 2007).

Dammert $(2005,2006,2008)$ sostiene que los medios tuvieron una importante influencia en la generación de temor durante la década de los 90, pero que hoy no es clara la vinculación entre los contenidos de los medios y el temor. La más reciente investigación en busca de los factores determinantes del temor concluyó que son indicadores relevantes en Chile la victimización previa, el nivel socioeconómico, el sexo y la edad, pero no se incorporó en el modelo de análisis el consumo de medios (Varela y Schwaderer, 2010).

En el presente trabajo buscamos llenar algunas de las numerosas lagunas que aún tiene la investigación sobre la relación entre el consumo de medios de comunicación y el temor al delito en Chile. En particular, abordamos la incidencia que el consumo de contenidos informativos de diversos medios de comunicación (televisión abierta, televisión de pago, diarios y radio) puede tener sobre la sensación de temor. Además 
se analiza, por separado, el vínculo entre el consumo de información en los distintos canales de televisión abierta y el miedo a ser víctima de un delito. Esto, con el objetivo de analizar si la diferencia en los contenidos que transmite cada canal tiene un impacto distinto en las percepciones de temor de los usuarios de medios.

Finalmente, y en una perspectiva distinta, también testeamos si en Chile existen evidencias de Agenda Setting en la materia. Es decir, si una mayor exposición al material informativo de los medios, especialmente a los contenidos sobre delincuencia, incide en que las personas consideren que las temáticas sobre seguridad ciudadana son de gran relevancia en la vida social.

\section{Fuentes y Metodología}

Para abordar los objetivos de este trabajo utilizamos los datos de la encuesta Jóvenes, Participación y Consumo de Medios, realizada por la Escuela de Periodismo de la Universidad Diego Portales y Feedback. Se trata de un estudio de opinión pública, de carácter probabilístico, que se realizó en los tres principales centros urbanos de Chile entre el 19 de agosto y el 6 septiembre de 2011 (Periodismo UDP-Feedback, 2011).

El muestreo de esta investigación se dividió tres estratos: Gran Santiago (631 casos), Gran Valparaíso (545 casos) y Gran Concepción (561 casos). Al interior de cada uno ellos, los individuos encuestados fueron seleccionados en tres etapas sucesivas: 1) sorteo aleatorio de manzanas o bloques (las unidades territoriales más pequeñas en que se agrupan las viviendas en Chile); 2) sorteo aleatorio de viviendas al interior de las manzanas o bloques; 3 ) sorteo aleatorio de una persona al interior de las viviendas. Los resultados obtenidos fueron ponderados por sexo, edad y región, de acuerdo a las proyecciones poblaciones del Instituto Nacional de Estadísticas para 2011. La muestra estuvo compuesta por 1.737 personas mayores de 18 años. Este tamaño, y el hecho que el estudio sea probabilístico, permitió hacer inferencias al universo de estudio con un error máximo de $2,4 \%$, bajo los supuestos de muestreo aleatorio simple, varianza máxima y un nivel de confianza de $95 \%$.

Es necesario aclarar que, pese a que la encuesta utilizada aborda preferentemente áreas temáticas vinculadas a la población juvenil, en la versión de 2011 se incluyó una numerosa muestra de mayores de 29 años, lo que también permite obtener conclusiones para el total de la población mayor de 18 años.

En la encuesta, el grado de temor a la delincuencia fue medido a través de la siguiente pregunta: "Actualmente, ¿Cuánto temor siente usted de ser víctima de un delito?", interrogante que tenía como alternativas de respuesta Nada (1), Poco (2), Bastante (3) y Mucho (4). La pregunta fue utilizada como la variable dependiente de un análisis de regresión que busca explicar los factores que están relacionados con sentir un mayor temor de ser víctima de un delito, especialmente para establecer el vínculo que el consumo de medios de comunicación puede tener con esta situación.

Como se observa en el Gráfico 1, los individuos con bastante o mucho temor a ser víctimas de un delito ascienden a $65 \%$, mientras que aquellos que tienen poco o nada de miedo llegan a $35 \%$. En esta escala progresiva de temor, el promedio de las respuestas fue de 2,9 (DE: 1,05). 


\section{ACTUALMENTE ¿CUÁNTO TEMOR SIENTE USTED A SER VÍCTIMA DE UN DELITO?}

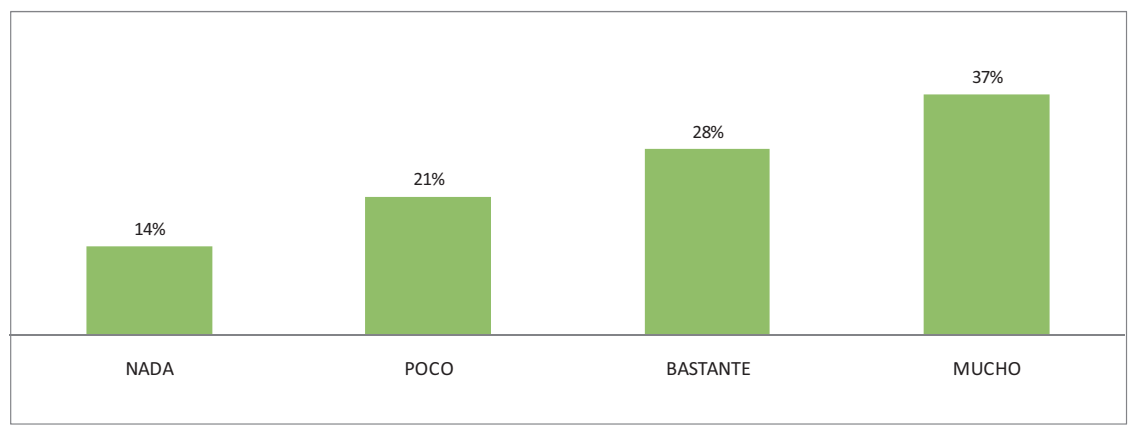

Gráfico 1. Temor a la delincuencia

Debido a las características de la variable dependiente -se trata de una escala ordinal, que no permite medir la distancia entre las distintas categorías de respuesta-, se optó por realizar un análisis de regresión ordinal, desechando la posibilidad de una regresión lineal múltiple, método más adecuado para variables de intervalo.

En el análisis se incorporaron variables independientes o explicativas relacionadas con el consumo de medios de comunicación, además de variables relacionadas con tres modelos explicativos que investigaciones anteriores han planteado frente al temor a la delincuencia (Allende, 2004). El Modelo de Vulnerabilidad propone que ciertas indicadores personales (como el sexo y la edad) y sociales (situación socioeconómica) hacen que las personas se sientan más expuestas e indefensas frente a los delitos, lo que se traduce en un mayor nivel de temor. El Modelo Victimización sostiene que las personas que han sido víctimas en el pasado tienen un mayor nivel de temor que el resto de la población, situación que se extendería a aquellos que conocen a individuos cercanos que han sido víctimas, lo que se denomina victimización vicaria. Finalmente, el Modelo de Control Social argumenta que las personas que perciben orden y seguridad en su entorno tienden a desarrollar un menor temor a ser víctimas de delitos.

Para medir si los medios de comunicación influyen sobre el temor a ser víctima de un delito, se incorporaron en el análisis distintas variables como la cantidad de horas diarias de noticias que las personas consumen, la cantidad de programas con alto contenido de delincuencia que declararon haber visto durante los últimos siete días y la frecuencia con que comentan las noticias sobre delincuencia.

En el caso de los contenidos informativos, se utilizó el promedio diario de horas de noticiarios que los encuestados declararon haber consumido en televisión abierta $(\mathrm{M}=2,0 ; \mathrm{DE}=2,12)$, televisión de pago $(\mathrm{M}=0,64 ; \mathrm{DE}=1,47)$, radio $(\mathrm{M}=0,98 ; \mathrm{DE}=$ $1,75)$, diarios impresos $(\mathrm{M}=0,40 ; \mathrm{DE}=0,96)$.

Debido a que la televisión abierta es el principal medio de comunicación que usan los chilenos para informarse (CNTV, 2011) y a que la cantidad de noticias sobre delincuencia que se emite en cada canal de televisión presenta variaciones relevantes 
(CNTV, 2006) ${ }^{1}$, se optó también por utilizar como variables independientes las horas de consumo de noticias en televisión abierta, pero separándolas por el canal de televisión en que las personas declaran informarse preferentemente. Esta separación permite saber si el consumo de noticias en distintos canales de televisión tiene un impacto diferenciado de acuerdo a los contenidos que éstos trasmiten.

Junto con las noticias, otro elemento relevante en la precepción de temor pueden ser los programas que se dedican exclusiva, o preferentemente, a temas de seguridad ciudadana. Para medir este efecto se consultó a los encuestados si habían visto, durante los últimos siete días, seis programas de este tipo que se trasmitían efectivamente al momento de hacer la encuesta. Con las respuestas se construyó un contador que tiene como valor mínimo 0 y máximo $6(\mathrm{M}=2,8 ; \mathrm{DE}=2,0)$.

Finalmente, se incorporó el impacto que pueden tener sobre el temor las conversaciones motivadas por noticias sobre la delincuencia con la familia, vecinos, compañeros de trabajo o estudio y amigos. Se creó un índice, con valor mínimo de 1 y máximo de 5 , considerando la frecuencia de este tipo de conversaciones $(\mathrm{M}=3,3$; $\mathrm{DE}=1,2$; Alpha de Cronbach $=0,81$. La ventaja de esta variable es que permite incorporar en el análisis si los medios de comunicación se relacionan con el temor al delito de forma más compleja e indirecta.

- Modelo de vulnerabilidad (física y social): La edad fue incorporada como una variable continua $(\mathrm{M}=42,9 ; \mathrm{DE}=17)$, mientras que el sexo fue tratado como una dicotómica (Mujeres $=52 \%$ ). En tanto, la clasificación de nivel socioeconómico se construyó utilizando el método de la Asociación Mundial de Investigación de Mercado (Esomar). Se trata de una metodología que combina el nivel educacional del jefe de hogar y la posesión de ciertos bienes materiales, operación que otorga una puntaje entre 0 y 1000 a cada hogar y permite ubicarlo en una escala socioeconómica. Esta escala está compuesta por los grupos A, B, C1, C2, C3, D y E. En este estudio se han agrupados los segmentos A, B y C1 (formando el grupo de altos ingresos) y los segmentos D y E (sector de menores ingresos). El resto corresponde a categorías intermedias entre ambos.

- Modelo de Victimización: Para medir la victimización previa se creó una variable dicotómica en que 1 significa haber sido víctima de algún tipo de delito durante los últimos doce meses, mientras 0 implica no haberlo sido ( $\mathrm{SI}=23,1 \%)$. Esta variable se construyó tras preguntar por cinco tipos de robos distintos: con violencia o intimidación en la calle, sin violencia o intimidación en la calle, con violencia o intimidación en el hogar, sin violencia o intimidación en el hogar y robo de vehículos.

En el caso de la victimización vicaria también se creó una variable dicotómica en que 1 significa tener algún miembro del hogar, vecino o conocido que ha sido víctima de de un delito en los últimos doce mes y 0 no conocer a nadie en esta situación $(\mathrm{SI}=48,3 \%)$. Para construir esta variable se utilizaron los mismos cinco tipos de robos usados en el caso de la victimización previa.

1 De acuerdo a una medición realizada por el Consejo Nacional de Televisión en 2006, los dos canales de televisión con mayor audiencia cubrían este frente noticioso de forma distinta. Mientras Chilevisión destinaba 19,9\% del tiempo de sus noticiarios a temas policiales, Televisión Nacional de Chile sólo otorgaba 9,9\% (CNTV, 2006). 
- Modelo de Control social: Como parte de esta hipótesis se calculó un índice de seguridad en el entorno, a partir de cuán seguros consideran las personas consideran su hogar, su barrio, su ciudad y su país. Las alternativas de respuesta que tenían las personas en cada una de estos casos eran: Nada (1), Poco (2), Bastante (3) y Mucho (4). El índice tuvo valor mínimo 1 y máximo $4(\mathrm{M}=2,57 ; \mathrm{DE}=0,66$; Alpha de Cronbach=0,81)

Por último, para poder estudiar si el consumo de contenidos mediales sobre delincuencia tiene impacto en que las personas consideren que la seguridad ciudadana es un tema de gran importancia (Agenda Setting), se construyó una segunda variable dependiente. Como base de ésta se utilizó la pregunta abierta de la encuesta Jóvenes, Participación y Consumo de Medios: "A su juicio, ¿cuál es el principal problema que afecta a los chilenos? ¿y en segundo lugar?". Las respuestas a estas dos preguntas se recodificaron creando una variable binaria en que se asignó el valor 1 a todos los que señalaron la delincuencia (o temas muy semejantes) como uno de los dos principales problemas del país y valor 0 al resto de la población $(1=20,9 \%)$. Por tratarse de una variable dependiente dicotómica, en este caso se optó por hacer una regresión logística.

\section{Desarrollo}

El análisis de regresión ordinal sobre los datos descritos nos permitó analizar, en primer lugar, el impacto del consumo de medios de comunicación -junto a otras variables relevantes-, sobre el temor al delito (Tablas 1 y 2), así como la influencia de los medios en la construcción de la agenda pública, particularmente en el área de seguridad ciudadana. Los resultados muestran que existe una relación positiva entre el uso de algunos medios de comunicación y tener un mayor nivel de temor a ser víctima de un delito. Particularmente, ver una mayor cantidad de horas de noticias en televisión abierta se vincula con presentar un grado superior de temor, lo que no ocurre al consumir contenidos informativos en otros medios como televisión de pago, radio y diarios.

Tabla 1. Regresión ordinal para predecir el temor a ser víctima de un delito ${ }^{2}$

\begin{tabular}{|l|c|c|}
\hline \multicolumn{3}{|c|}{${ }^{*} \mathrm{p}<0,05 ;{ }^{*} \mathrm{p}<0,01$} \\
\hline Sexo (Hombre) & $\mathbf{E}$ & Wald \\
Mujer & $0,612 * *$ & 19,066 \\
\hline Edad & $0,019 * *$ & 29,664 \\
\hline NSE (DE) & $-0,012$ & 0,004 \\
ABC1 & $-0,028$ & 0,031 \\
C2 & $-0,162$ & 1,588 \\
C3 & $0,113^{* *}$ & 15,866 \\
\hline Horas diarios de noticias en Televisión & 0,038 & 0,762 \\
\hline Hoierta & $-0,004$ & 0,014 \\
\hline pago diarias de noticias en Televisión de & $-0,050$ & 0,890 \\
\hline Horas diarias de noticias en Radios & & \\
\hline Horas diarias de noticias en Diarios &
\end{tabular}

2 En este modelo regresión ordinal los coeficientes negativos están asociados con una disminución del temor a la delincuencia, en tanto los coeficientes positivos están vinculados con un alza del temor (Tabla 1) 


\begin{tabular}{|l|c|c|}
\hline $\begin{array}{l}\text { Ver programas con alto contenido sobre } \\
\text { delincuencia en último siete días }\end{array}$ & $-0,016$ & 0,336 \\
\hline $\begin{array}{l}\text { Conversaciones interpersonales respecto a } \\
\text { noticias sobre delincuencia }\end{array}$ & $0,150^{* *}$ & 9,973 \\
\hline $\begin{array}{l}\text { Victimización previa directa } \\
\text { Sí }\end{array}$ & $0,612^{* *}$ & 20,649 \\
\hline $\begin{array}{l}\text { Victimización vicaria } \\
\text { Sí }\end{array}$ & 0,008 & 0,006 \\
\hline Percepción de seguridad en el entorno & $-0,937 * *$ & 114,531 \\
\hline Pseudo R-cuadrado Nagelkerke & 0,179 & \\
\hline
\end{tabular}

Junto a este impacto directo, se observa que también hay una relación entre el miedo a los delitos y la frecuencia con que las personas conversan con otros respecto a noticias sobre delincuencia. Esto da cuenta de que la incidencia de los medios se puede producir de forma indirecta y más compleja, interactuando con otras variables y formas de comunicación. Por ello, pese a que los resultados del análisis de regresión muestran una incidencia de los medios estadísticamente significativa, es importante contextualizar esta situación. Hay otras variables que, de acuerdo a los índices de Wald del modelo, tienen una incidencia mayor.

Según los resultados obtenidos, la variable con mayor impacto es la percepción de seguridad en el entorno: mientras más alta es la percepción de seguridad más bajo es el temor a la delincuencia. Adicionalmente, el sexo y la edad son factores relacionados con la variable dependiente. Ser mujer y cada año adicional de los entrevistados incrementa la probabilidad de que sientan miedo de ser víctimas de un delito. En tanto, las características socioeconómicas no muestran tener una incidencia estadísticamente significativa. Finalmente, en este primer modelo, la victimización previa (haber sido víctima de un delito durante los últimos doce meses), sí es un predictor de mayores niveles de temor. No así la victimización vicaria.

Debido a que la información disponible muestra que la proporción de noticias sobre delincuencia difiere entre los distintos canales de televisión abierta (CNTV, 2011), en un segundo modelo de regresión introdujimos por separado cuatro variables cuantitativas que identificaban la cantidad de horas de consumo y la estación televisiva en que las personas declararon preferir informarse. Se utilizaron los cuatro principales canales de de televisión abierta que funcionan en Chile: Chilevisión (propiedad privada), Televisión Nacional de Chile (estatal), Canal 13 (propiedad mixta entre privados y la Iglesia Católica) y Mega (privado). Los resultados muestran que el consumo de noticias en Chilevisión es el único que tiene un impacto positivo en el temor. No sucede lo mismo con ninguno de los otros tres (Ver Tabla 2).

Tabla 2. Regresión ordinal para predecir el temor a ser víctima de un delito (segundo modelo) ${ }^{*} \mathrm{p}<0,05 ;{ }^{* *} \mathrm{p}<0,01$

\begin{tabular}{|l|c|c|}
\hline & E & Wald \\
\hline $\begin{array}{l}\text { Sexo (Hombre) } \\
\text { Mujer }\end{array}$ & $0,518^{* *}$ & 25,726 \\
\hline Edad & $0,018^{* *}$ & 30,648 \\
\hline
\end{tabular}




\begin{tabular}{|l|c|c|}
\hline NSE (DE) & 0,108 & 0,286 \\
ABC1 & 0,013 & 0,008 \\
C2 & $-0,064$ & 0,272 \\
C3 & $0,090^{* *}$ & 10,548 \\
\hline Chilevisión & $-0,072$ & 3,778 \\
\hline Televisión Nacional & 0,028 & 0,288 \\
\hline Canal 13 & 0,037 & 0,389 \\
\hline Mega & $-0,005$ & 0,033 \\
\hline $\begin{array}{l}\text { Ver programas con alto contenido sobre } \\
\text { delincuencia en último siete días }\end{array}$ & $0,168^{* *}$ & 13,629 \\
\hline $\begin{array}{l}\text { Conversaciones interpersonales respecto a } \\
\text { noticias sobre delincuencia }\end{array}$ & $0,521^{* *}$ & 16,391 \\
\hline Victimización previa directa & $-0,090$ & 0,710 \\
Sí & $-0,967^{* *}$ & 133,289 \\
\hline Victimización vicaria & 0,188 & \\
Sí & \\
\hline Percepción de seguridad en el entorno & \\
\hline Pseudo R-cuadrado Nagelkerke &
\end{tabular}

Este resultado es relevante, ya que un análisis de contenido de los noticieros centrales de estos canales, realizado por Conecta Media Research, mostró que Chilevisión es la estación que más tiempo dedica a los noticias "policiales" o relacionadas con la delincuencia. Este canal dedicó el 18\% de su programación noticiosa a cubrir la delincuencia, lo que se tradujo en 402 minutos en 30 días. En promedio, los otros tres canales dedicaron $12 \%$ de su tiempo a este tema (Ver Gráfico 2). Los datos de esta medición corresponden a los contenidos transmitidos entre el 18 de julio y el 18 de agosto de 2011, justo el mes previo a que se realizara el trabajo de campo de la encuesta.En este segundo modelo, las demás variables se comportaron de forma muy similar al anterior.

\section{PORCENTAJE DE MINUTOS DEDICADOS A DELINCUENCIA EN NOTICIARIOS}

\section{CENTRALES (18 DE JULIO- 18 DE AGOSTO DE 2011)}

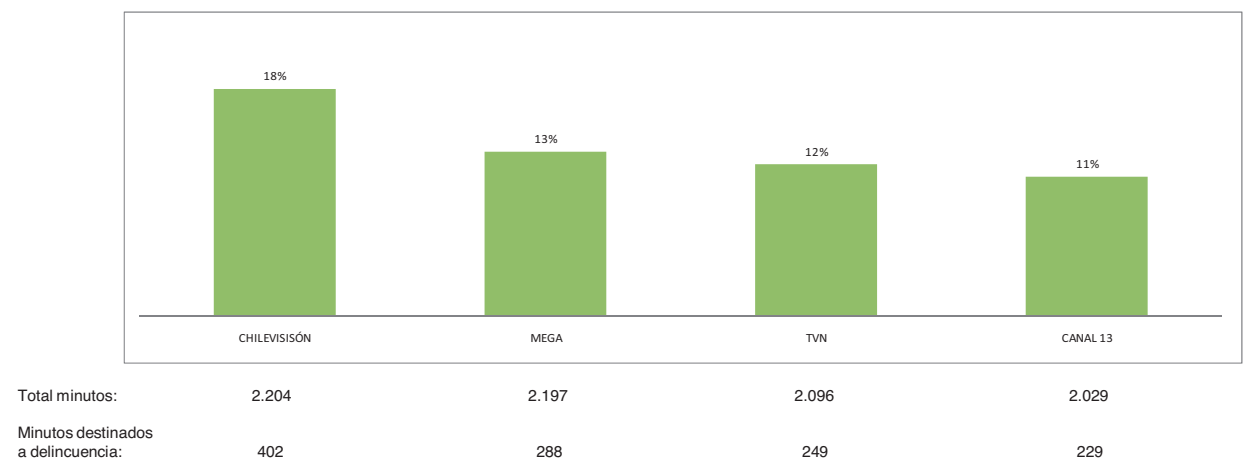

Gráfico 2. Cobertura de delincuencia por canal. Fuente: Conecta Media Research

Por último, se buscó testear si ver contenidos sobre delincuencia influyen en que las personas consideren que esta materia es uno de los principales problemas del país 
(Agenda Setting). El análisis mostró que efectivamente exponerse a una mayor cantidad de programas que abordan temas de seguridad ciudadana está vinculado con pensar que el tema es uno de los más importantes (Ver Tabla 3).

También mostraron tener una relación significativa la edad (cada año adicional incrementa la probabilidad de que las personas consideren que es un asunto relevante para el país) y la percepción de seguridad en el entorno (mientras más seguros se sienten los encuestados, menos relevancia le asignan al tema). A diferencia de la relación con el temor, la victimización previa y el sexo no muestran un vínculo significativo.

Tabla 3. Regresión logística para predecir efecto de Agenda Setting ${ }^{3}$ ${ }^{*} \mathrm{p}<0,05 ;{ }^{*} \mathrm{p}<0,01$

\begin{tabular}{|l|l|l|}
\hline & \multicolumn{1}{|c|}{$\operatorname{Exp(B)}$} & \multicolumn{1}{|c|}{ Wald } \\
\hline $\begin{array}{l}\text { Sexo (Hombre) } \\
\text { Mujer }\end{array}$ & 0,149 & 1,232 \\
\hline Edad & $0,018^{* *}$ & 17,732 \\
\hline $\begin{array}{l}\text { NSE (DE) } \\
\text { ABC1 }\end{array}$ & -0321 & 1,177 \\
C2 & 0,251 & 1,681 \\
C3 & $-0,060$ & 0,133 \\
\hline $\begin{array}{l}\text { Ver programas con alto contenido sobre } \\
\text { delincuencia en último siete días }\end{array}$ & $0,129^{* *}$ & 13,523 \\
\hline $\begin{array}{l}\text { Conversaciones interpersonales } \\
\text { respecto a noticias sobre delincuencia }\end{array}$ & $-0,036$ & 0,359 \\
\hline $\begin{array}{l}\text { Victimización previa directa } \\
\text { Sí }\end{array}$ & 0,084 & 0,255 \\
\hline $\begin{array}{l}\text { Victimización vicaria } \\
\text { Sí }\end{array}$ & 0,142 & 0,981 \\
\hline Percepción de seguridad en el entorno & $-0,422^{* *}$ & 16,143 \\
\hline Pseudo R-cuadrado Nagelkerke & 0,061 & \\
\hline
\end{tabular}

\section{Conclusiones}

La influencia del consumo de medios de comunicación sobre el temor a ser víctima de un delito es una línea de investigación que ha generado, a nivel mundial, resultados disímiles y que están lejos de generar consenso en el mundo académico.

Pese a la fuerte brecha que existe en Chile entre las tasas efectivas de delitos (más bien moderadas) y los altísimos niveles de temor que presenta la población, se trata de un tema que ha tenido un escaso desarrollo en el país.

El objetivo de esta investigación no es plantear que los niveles de temor se explican sólo por la exposición a los medios, como si estos tuviesen un efecto automático en audiencias pasivas. Lo que se busca es entender si los medios de comunicación juegan un rol en este proceso junto a una serie de variables del mundo real, como las

${ }^{3}$ En este modelo de regresión logística, los $\operatorname{Exp}(\mathrm{B})$ menores a 1 disminuyen la chance de que las personas consideren que la delincuencia es uno de los dos temas más importantes del país, mientras que los $\operatorname{Exp}(\mathrm{B})$ incrementan esa posibilidad. 
experiencias previas de victimización y las características sociodemográficas de la población. Por eso, de la misma manera que pensamos que sería un error explicar el temor exclusivamente a partir de la exposición a los medios, creemos que también lo sería negar ex ante que tengan alguna incidencia.

De acuerdo a los resultados de la presente investigación, el único medio relacionado positivamente con la percepción de temor es la televisión; todos los demás no muestran tener incidencia. Este vínculo exclusivo entre las noticias en televisión abierta y el temor se podría explicar por la profusa cobertura que, a diferencia de los otros, este medio realiza respecto a la delincuencia (Dastres, 2003). A lo que se suma la espectacularidad con que son mostrados y narrados este tipo de episodios.

El impacto de la televisión abierta se produce a través de dos vías. La primera la denominaremos directa: mientras más horas de noticias en televisión consumen las personas, se incrementa el nivel de miedo a la delincuencia. Sin embargo, el efecto de los diferentes canales sobre el temor no es parejo. De hecho, la relación sólo es positiva en uno de ellos, aquel que transmite la mayor cantidad de programación sobre delincuencia.

La segunda forma de incidencia de la televisión abierta es indirecta o mediada: en la medida en que las personas sostienen con mayor frecuencia conversaciones que se originan en noticias sobre delitos, aumenta su nivel de temor. Este hallazgo se entronca con una larga línea de investigación iniciada por Lazarsfeld y Katz: la teoría de la Influencia Personal o el flujo de la comunicación en dos escalones. Enmarcados en la línea de efectos limitados de los medios, estos autores plantearon que el principal impacto de los medios sobre la audiencia no se produce de forma directa. Según ellos, es en las conversaciones interpersonales posteriores a la recepción de los mensajes mediales (segundo escalón de la comunicación), donde la influencia es mayor (Lazarsfeld y Katz, 1955).

Estos hallazgos no significan que otras variables, asociadas al mundo real o las características personales, no sean relevantes. Por el contrario, los análisis de regresión mostraron que pese a tener una relación significativa, los medios de comunicación son bastante menos importantes que otras variables al momento de explicar el temor al crimen. Es así como los llamados modelos de victimización, vulnerabilidad y control social, recibieron considerable apoyo de la evidencia empírica.

En el caso del modelo victimización, haber sido víctima de un delito durante los últimos doce meses (victimización previa) mostró estar vinculado con el temor, lo que no sucede en el caso de los entrevistados que tienen familiares o amigos que han sido víctimas (victimización vicaria). En tanto, el modelo de vulnerabilidad también recibió respaldo, sobre todo respecto a la denominada vulnerabilidad física: ser mujer y pertenecer a los grupos etarios mayores se encuentra relacionado con un aumento de la percepción de temor, a diferencia de lo que sucede con la pertenencia a los grupos socioeconómicos bajos (vulnerabilidad social). Finalmente, el modelo de control social mostró una importante capacidad explicativa, siendo la percepción de seguridad en el entono otro factor determinante del miedo al delito.

En términos de mayor a menor influencia, la variable más relevante resultó ser la percepción de seguridad en el entorno, seguida de la edad, la victimización previa y 
el sexo. Sólo después aparecen las variables asociadas al consumo de medios. Adicionalmente, la investigación confirmó hallazgos anteriores en torno a la influencia de los medios en la construcción de la agenda pública sobre seguridad ciudadana.

Nos parece que los datos arrojados por la investigación muestran que se debe considerar, y seguir investigando, el papel que juegan los medios en la percepción del temor, especialmente en casos en que existe una fuerte brecha entre la victimización real y el miedo a la delincuencia. Si bien no son los únicos elementos relevantes, claramente deben ser parte del análisis en la explicación del fenómeno.

Por último, sería conveniente que investigaciones futuras pudieran medir el impacto de los medios a través de encuestas de panel, método que permitiría establecer con mayor claridad si un cambio en el consumo de noticias en un mismo individuo tiene efectos sobre sus niveles de temor.

\section{Referencias bibliográficas}

ALLENDE, Catalina (2004): "El peso del temor a la delincuencia y sus factores determinantes en la población urbana chilena". Primer Simposio Nacional de Investigación sobre violencia y delincuencia. ISUC/FPC. pp. 199-220.

BROWNE, Magdalena y TOMICIC, Visjna (2007): "Crimen y temor: el rol de los medios". Cuadernos de Información, $\mathrm{n}^{\circ}$ 20, disponible en: http://www.redalyc.org /src/inicio/ArtPdfRed.jsp?iCve=97120369003. (Enero-julio 2007). pp 21-36

CENTRO DE ESTUDIOS PÚBLICOS (2011): Estudio Nacional de opinión Pública junio-julio 2011, Chile. (http://www.cepchile.cl/dms/lang_1/doc_4844.html).

CONSEJO NACIONAL DE TELEVISIÓN (CNTV): Departamento de Estudios (2006). Seguridad Ciudadana en Noticiarios de TV Abierta (www.cntv.cl).

CONSEJO NACIONAL DE TELEVISIÓN (CNTV): Departamento de Estudios (2011). Análisis Noticiarios (www.cntv.cl).

DAMMERT, Lucía (2005):"Violencia en los medios de comunicación, generación noticiosa y percepción ciudadana". CERBINO, Mauro (Ed.): Violencia, miedos y medios de comunicación: desafíos y oportunidades Ecuador. Flacso, pp 51-72.

DAMMERT, Lucía y MALONE, Mary (2006): "Fear does it take a village? Policy strategies and fear of crime in Latin America". Latin American Politics and Society, Vol.48.

DAMMERT, Lucía; RUZ, Felipe; y SALAZAR, Felipe (2008): ¿Politicas de seguridad a ciegas?: desafios para la construcción de sistemas de información en América Latina. Flacso, Chile.

DASTRES, Cecilia; SPENCER, Cristian; MUZZOPAPPA, Eva; y SAÉZ, Chiara (2005): "La Construcción de Noticias sobre Seguridad Ciudadana en prensa Escrita y Televisión. ¿Posicionamiento, Distorsión o Comprensión?” Colección Seguridad Ciudadana y Democracia. Santiago.

DITTON, Jason; CHADEE, Derek; FARRALL, Stephen; GILCHRIST, Elizabeth; and BANNISTER, Jon (2004): "From Imitation to Intimidation: A Note on the Cu- 
rious and Changing Relationship between the Media, Crime and Fear of Crime". British Journal of Criminology 4, pp 595-610.

DOWLER, Kenneth (2003): "Media consumption and public attitudes toward crime and justice: the relationship between fear of crime, punitive attitudes, and perceived police effectiveness". Journal of Criminal Justice and Popular Culture, 10(2), pp 109-126.

ESCUELA DE PERIODISMO UNIVERSIDAD DIEGO PORTALES-FEEDBACK (2011): Encuesta de Jóvenes, Participación y Consumo de Medios (www.udp.cl).

FUNDACIÓN PAZ CIUDADANA-ADIMARK GFK (2012): Serie de datos para comunas del pais (http://www.pazciudadana.cl/docs/pub_20120309124646.pdf).

FUNDACIÓN PAZ CIUDADANA (2010): Balance de la Delincuencia 2010 (http://www.pazciudadana.cl/docs/pub_20110607103119.pdf).

GERBNER, George; GROSS, Larry; MORGAN, Michael; and SIGNORIELLI, Nancy (1980): "The mainstreaming of America: Violence Profile" 11, Journal of Communication. 30, pp 10-29

LAPOP (2010): The Americas Barometer by the Latin American Public Opinion Project, (www.LapopSurveys.org).

LAZARSFELD, Paul; KATZ, Elihu. (1955): Personal Influence. New York. Free Press.

MCCOMBS, Maxwell (1996): "Influencia de las Noticias sobre nuestras imágenes del mundo". En BRYANT, Jennings y ZILLMAN, Dolf (eds.): Los efectos de los medios de comunicación. Investigación y teorías. Barcelona, Paidós.

MINISTERIO DEL INTERIOR Y SEGURIDAD PÚBLICA (2010): Encuesta Nacional de Seguridad Ciudadana (ENUSC). http://www.seguridadpublica.gov.cl /files/resultados_pais_enusc_2010_ms_05abr2011.pdf)

REINER, Robert (2007): "Media made Criminality: The representation of Crime in the Mass Media". En MAGUIRE, Mike; MORGAN, Rod and REINER, Robert (eds.): The Oxford handbook of criminology. Oxford University Press, Oxford, UK, pp. 302-337.

STUTMAN, Suzanne (1995): An opportunity to Prevent Violence: The Role of the Media. Mimeo.

VARELA, Fernanda; SCHWADERER, Hermann (2010): Determinantes del Temor al Delito en Chile. Santiago, Chile, Fundación Paz Ciudadana. 